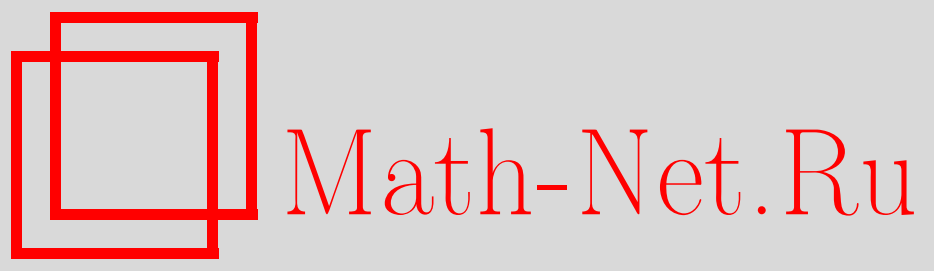

В. И. Кляцкин, Аномальные волны как объект статистической топографии. Постановка задачи, ТМФ, 2014, том 180, номер 1, 112-124

DOI: https://doi.org/10.4213/tmf8656

Использование Общероссийского математического портала Math-Net.Ru подразумевает, что вы прочитали и согласны с пользовательским соглашением http: //www . mathnet.ru/rus/agreement

Параметры загрузки:

IP: 54.237 .206 .68

26 апреля 2023 г., 08:57:24

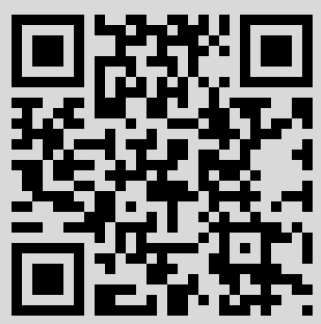




\section{АНОМАЛЬНЫЕ ВОЛНЫ КАК ОБЪЕКТ СТАТИСТИЧЕСКОЙ ТОПОГРАФИИ. ПОСТАНОВКА ЗАДАЧИ}

На основе идей статистической топографии анализируется краевая задача о возникновении на морской поверхности аномально больших волн (волн-убийц, или rogue waves). Краевое условие для морской поверхности рассматривается как замкнутое стохастическое квазилинейное уравнение в кинематическом приближении. Получено стохастическое уравнение Лиувилля, являющееся основой для вывода уравнения, описывающего совместную плотность вероятностей полей смещения морской поверхности и его градиента. Формулируется статистическая задача с учетом стохастических топографических неоднородностей морского дна, описывающая диффузию в фазовом пространстве, решение которой должно ответить на вопрос о том, содержится ли в рассматриваемом квазилинейном уравнении информация о существовании аномально больших волн.

Ключевые слова: аномальные волны, волны-убийцы, уравнение Лиувилля, статистическая топография.

DOI: $10.4213 / \operatorname{tmf} 8656$

\section{1. ВВЕДЕНИЕ. О СТОХАСТИЧЕСКОМ СТРУКТУРООБРАЗОВАНИИ В СЛУЧАЙНЫХ СРЕДАХ}

Вопрос о стохастическом структурообразовании в случайных средах волнует человечество с античных времен (см., например, труд [1]). Не составляют исключения и наши времена. При этом рассматриваются в основном два типа задач: о структурообразовании в случайной среде инородной материи (кластеризация) и структурообразование само́й случайной среды. Первый тип структурообразования связан с параметрическим возбуждением различных физических полей в случайных средах. Такие задачи имеют место в гидродинамике (пассивная скалярная примесь в турбулентном потоке), в магнитной гидродинамике (пассивная векторная примесь - магнитное поле в турбулентном потоке) и распространение волн различной

${ }^{*}$ Институт физики атмосферы им. А. М. Обухова РАН, Москва, Россия. E-mail: klyatskin@yandex.ru 
природы (акустические, радиоволны, свет и лазерное излучение) в случайных средах (см., например, монографии [2], [3]). Отметим, что еще в прошлом веке было хорошо известно о возможности возникновения стохастической неустойчивости (параметрического возбуждения) в различных динамических системах за счет флуктуаций параметров самой системы. Но вплоть до 80-х гг. прошлого столетия никто этими вопросами практически не интересовался. Заслуга авторов работы [4] состоит в том, что они, по-видимому, первыми обратили внимание на возможность стохастического структурообразования благодаря подобному параметрическому возбуждению, которое также было известно в то время из наблюдений различного рода. Вопрос лишь в том, что́ они под этим термином подразумевали. В работе [4] подразумевалось, что “структуры, возникающие в случайной среде, имеют своеобразный характер: они имеют вид пиков, появляющихся в случайных местах и в случайные моменты времени. Промежутки между ними характеризуются малой интенсивностью и большой протяженностью". Однако это не соответствует действительности. В самом деле, реализация случайного процесса во времени для любой точки пространства имеет описанный вид, обусловленный логнормальным одновременнь́м распределением вероятностей, имеющим пологий "хвост". Большие, но редкие выбросы (флуктуации) обусловлены этими хвостами. При этом структурообразование происходит в физическом пространстве, в частности, для примера, рассмотренного в работе [4], структурообразование не осуществляется. Подробное обсуждение этих вопросов содержится в монографиях [2], [3] и в работах [5]-[8].

Что касается структурообразования само́й случайной среды, то такая ситуация может осуществляться даже в гауссовых случайных полях. Подобные явления имеют место в двумерных задачах геофизической гидродинамики во вращающейся жидкости со случайной топографией дна (см., например, работы [9]-[11]).

Простейший случай когерентного явления соответствует двумерному несжимаемому потоку идеальной жидкости на плоскости переменной $\mathbf{R}=(x, y)$. Этот поток описывается функцией тока $\psi(\mathbf{R}, t)$, которая удовлетворяет уравнению

$$
\frac{\partial}{\partial t} \Delta \psi(\mathbf{R}, t)=J\{\Delta \psi(\mathbf{R}, t), \psi(\mathbf{R}, t)\}, \quad \psi(\mathbf{R}, 0)=\psi_{0}(\mathbf{R}),
$$

где $J\{\psi(\mathbf{R}, t), \varphi(\mathbf{R}, t)\}$ - якобиан двух функций.

Нелинейное взаимодействие должно привести гидродинамическую систему (1) к статистическому равновесию. Учитывая, что процесс установления такого равновесия состоит из большого числа взаимодействий возмущений различных масштабов друг с другом, можно предположить, что в простейшем случае статистически однородного и изотропного случайного начального поля $\psi_{0}(\mathbf{R})$ такое равновесное распределение будет гауссовым, и задача состоит в определении его параметров. Случайная функция тока $\psi(\mathbf{R}, t)$ в процессе эволюции остается пространственно однородной и изотропной. С учетом того, что она определена с точностью до аддитивной постоянной, ее статистические характеристики описываются одновременно́й структурной функцией

$$
D_{\psi}\left(\mathbf{R}-\mathbf{R}^{\prime}, t\right)=\left\langle\left[\psi(\mathbf{R}, t)-\psi\left(\mathbf{R}^{\prime}, t\right)\right]^{2}\right\rangle=2\left[B_{\psi}(0, t)-B_{\psi}\left(\mathbf{R}-\mathbf{R}^{\prime}, t\right)\right],
$$

где через $B_{\psi}\left(\mathbf{R}-\mathbf{R}^{\prime}, t\right)=\left\langle\psi(\mathbf{R}, t) \psi\left(\mathbf{R}^{\prime}, t\right)\right\rangle$ обозначена пространственная корреляционная функция поля $\psi(\mathbf{R}, t)$. 
Если искать стационарное (равновесное) распределение в классе гауссовых распределений статистически однородных и изотропных полей $\psi(\mathbf{R}, t)$, которые описываются стационарной структурной функцией $D_{\psi}(R)=\lim _{t \rightarrow \infty} D_{\psi}(\mathbf{R}, t)$, то для нее можно получить уравнение [9]

$$
\left(\Delta_{q}+\lambda\right) \Delta_{q} D_{\psi}(q)=0,
$$

где $\Delta_{q}$ - радиальная часть оператора Лапласа, $q=\left|\mathbf{R}-\mathbf{R}^{\prime}\right|$, а постоянная разделения $\lambda$ имеет размерность квадрата обратной длины.

Имеются два возможных решения уравнения (2), соответствующие положительному и отрицательному значениям постоянной $\lambda$.

В случае $\lambda=k_{0}^{2}>0$ уравнение (2) сводится к уравнению

$$
\Delta_{q} D_{\psi}(q)=C J_{0}\left(k_{0} q\right)
$$

где $J_{0}(z)$ - функция Бесселя первого рода. В этом случае функция $D_{\psi}(q)$ определяется из решения уравнения Лапласа, и в результате мы получаем для спектральной плотности структурной функции выражение $E(k)=E \delta\left(k-k_{0}\right)$, что соответствует случайным структурам с определенным фиксированным пространственным масштабом. Такими структурами для данной задачи являются вихри, т. е. в этом случае имеет место структурообразование в виде вихрогенезиса.

Во втором случае $\lambda=-k_{0}^{2}<0$ соответствующая спектральная плотность структурной функции имеет вид

$$
E(k)=\frac{E_{0}}{k^{2}+k_{0}^{2}},
$$

что соответствует распределению Гиббса с двумя интегралами движения - интегралом энергии и квадратом вихря поля скорости.

Аналогичным образом можно рассмотреть равновесные состояния для квазигеострофических потоков (с учетом вращения) со случайной топографией подстилающей поверхности в рамках моделей однослойного и двуслойного потоков жидкости, где мы имеем аналогичную ситуацию.

В случае однослойной модели поток жидкости в двумерной плоскости переменной $\mathbf{R}=(x, y)$ описывает баротропное движение жидкости. В более общем случае для исследования бароклинных движений используется двуслойная модель гидродинамических потоков. При этом в двуслойной жидкости появляются уже два фиксированных размера. Подобные структуры наблюдались в экспериментальных исследованиях вращающейся жидкости (см., например, работы [12], [13] и монографию [15]), при численном моделировании сдвиговой неустойчивости [16], а также при наблюдении поля поверхностных течений в Балтийском море из космоса [17].

Отметим, что на поверхности морей и океанов время от времени наблюдаются аномально высокие волны, именуемые волнами-убийцами (см., например, монографии [18], [19] и лекции Захарова [20]). Это, конечно, также является примером стохастического структурообразования само́й случайной среды. Подобные волны возникают в случайных местах и в случайные моменты времени. Изучение причин и возможных механизмов возникновения таких стохастических структур в настоящее время привлекает многих исследователей (см., например, работы [21]-[24], в которых также приведена подробная библиография). Изучаются различные динамические модели на основе как численного моделирования, так и аналитических 

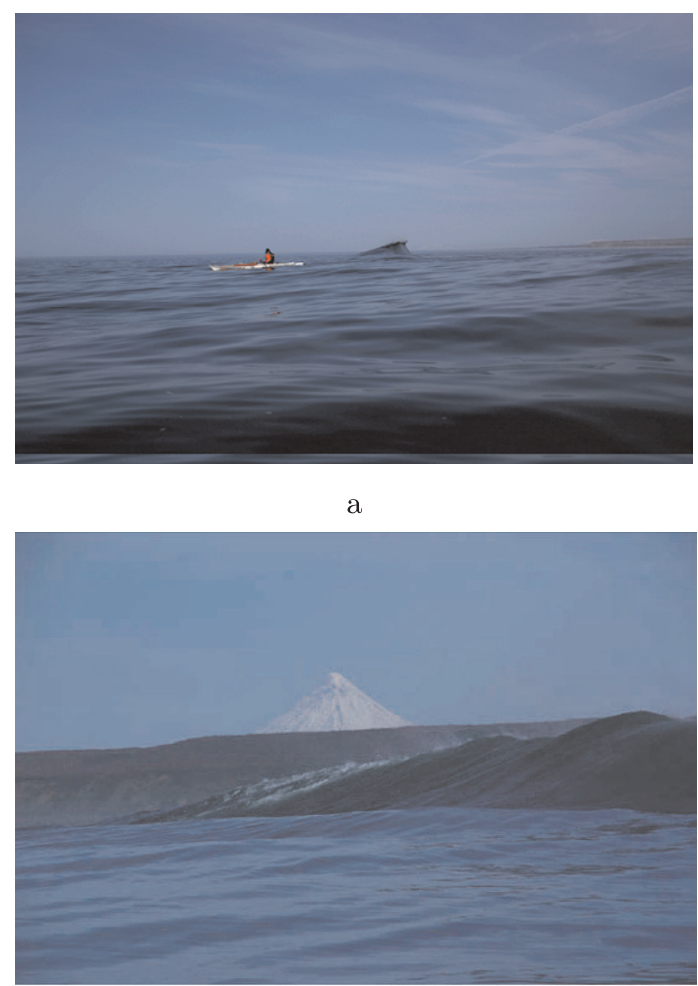

б

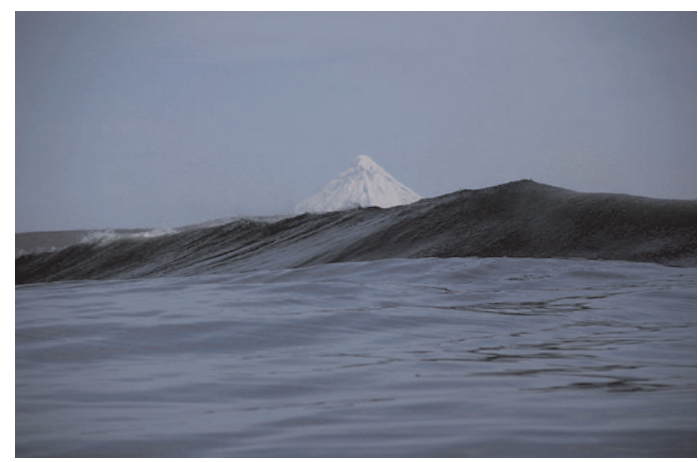

B

Рис. 1. Аномальная волна: вид сбоку (а), вид с фронта - начало волны (б) и середина волны (в).

результатов, связанных с нелинейным уравнением Шредингера. При этом зачастую при рассмотрении реализаций случайных процессов во времени именно появление редких, но больших выбросов отождествляется с волнами-убийцами, как это делалось ранее в указанной работе [4].

На рис. 1 приведены три фотографии необычной узкой, протяженной и неподвижной водной структуры высотой 4-5 м, которая наблюдалась 11 июня 2006 г. 


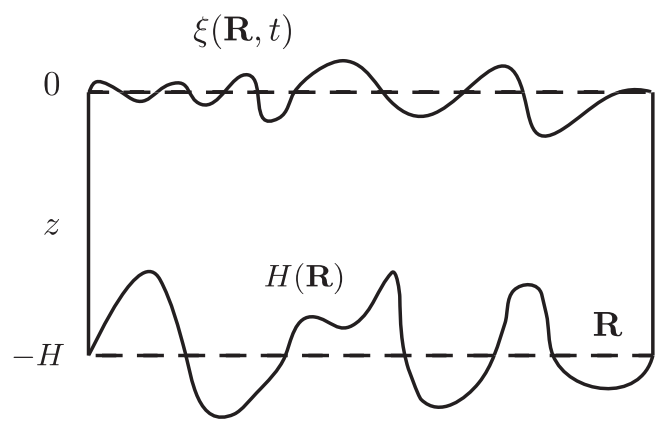

Рис. 2. Возмущение морской поверхности.

у Тихоокеанского побережья Камчатки в 1-1.5 км от берега, в 25 км севернее мыса Ольги. Обнаружено такое структурообразование было совершенно случайно во время месячной экстремальной экспедиции на каяках, длина которых составляет примерно 5 м. Отметим, что первый рисунок приводился ранее в упомянутых лекциях Захарова [20].

Вот как автор этих фотографий М. М. Соколовский описывал это явление в своем письме к родителям: “Это была, конечно, странная волна, так как она несколько раз повторялась, а потом исчезала. Вокруг никаких волн не наблюдалось - тишь да гладь".

В настоящей работе мы сформулируем и рассмотрим краевую задачу о возникновении аномально больших волн на морской поверхности на основе идей статистической топографии аналогично тому, как мы делали это раньше для стохастических задач с параметрическим возбуждением (см. монографии [2], [3] и работы [5]-[8]).

\section{2. ВОЛНЫ НА МОРСКОЙ ПОВЕРХНОСТИ}

Пусть $\mathbf{r}=\left\{r_{i}\right\}$ - трехмерная пространственная координата, здесь $i=1,2,3$. Обозначим через $z=r_{3}$ вертикальную координату, а через $R_{\alpha}, \alpha=1,2,-$ координаты в горизонтальной плоскости, перпендикулярной оси $z$. Тогда $\mathbf{r}=\{\mathbf{R}, z\}$. Соответственно трехмерное гидродинамическое поле скоростей $\mathbf{u}(\mathbf{r} ; t)$ представим в виде совокупности горизонтальной и вертикальной составляющих поля скорости, т. е. в виде

$$
u_{i}(\mathbf{r} ; t)=\left\{u_{\alpha}(\mathbf{R}, z ; t), w(\mathbf{R}, z ; t)\right\}, \quad i=1,2,3, \quad \alpha=1,2 .
$$

Смещение водной границы на морской поверхности задается как кинематическое краевое условие на поверхности $z=\xi(\mathbf{R}, t)$ (см. рис. 2), которое имеет вид

$$
\frac{d \xi(\mathbf{R}, t)}{d t}=\left.w_{z}(\mathbf{R}, z ; t)\right|_{z=\xi(\mathbf{R}, t)} .
$$

Здесь $\frac{d \xi(\mathbf{R}, t)}{d t}-$ полная производная смещения поверхности.

Краевое условие (3) можно рассматривать как замкнутое стохастическое квазилинейное уравнение в рамках кинематического приближения, т. е. при заданных статистических характеристиках полей скоростей $\mathbf{u}(\mathbf{R}, z ; t)$ и $w(\mathbf{R}, z ; t)$ :

$$
\frac{\partial \xi(\mathbf{R}, t)}{\partial t}+u_{\alpha}(\mathbf{R}, \xi(\mathbf{R}, t), t) \frac{\partial \xi(\mathbf{R}, t)}{\partial R_{\alpha}}=w_{z}(\mathbf{R}, \xi(\mathbf{R}, t) ; t)
$$


с начальным условием $\xi(\mathbf{R}, 0)=\xi_{0}(\mathbf{R})$. Уравнение $(4)$ при этом описывает генерацию волн на морской поверхности, вызванных вертикальной составляющей гидродинамического поля скоростей. Дифференцируя уравнение (4) по $\mathbf{R}$ для градиента смещения поверхности $p_{\beta}(\mathbf{R}, t)=\partial \xi(\mathbf{R}, t) / \partial R_{\beta}$, характеризующего наклон морской поверхности, получаем уравнение

$$
\begin{aligned}
\frac{\partial p_{\beta}(\mathbf{R}, t)}{\partial t}+ & \left(\left.\frac{\partial u_{\alpha}(\mathbf{R}, z ; t)}{\partial R_{\beta}}\right|_{z=\xi(\mathbf{R}, t)}+\frac{\partial u_{\alpha}(\mathbf{R}, \xi(\mathbf{R}, t) ; t)}{\partial z} p_{\beta}(\mathbf{R}, t)\right) p_{\alpha}(\mathbf{R}, t)+ \\
+ & u_{\alpha}(\mathbf{R}, \xi(\mathbf{R}, t), t) \frac{\partial p_{\alpha}(\mathbf{R}, t)}{\partial R_{\beta}}= \\
& =\left.\frac{\partial w_{z}(\mathbf{R}, z ; t)}{\partial R_{\beta}}\right|_{z=\xi(\mathbf{R}, t)}+\frac{\partial w_{z}(\mathbf{R}, \xi(\mathbf{R}, t) ; t)}{\partial z} p_{\beta}(\mathbf{R}, t)
\end{aligned}
$$

с начальным условием

$$
\mathbf{p}(\mathbf{R}, 0)=\mathbf{p}_{0}(\mathbf{R})=\frac{\partial \xi_{0}(\mathbf{R})}{\partial \mathbf{R}}
$$

Отметим, что для рассматриваемой задачи имеется и второе краевое условие, связанное с неоднородностями топографии морского дна (см. рис. 2). В рамках кинематического приближения это краевое условие проявляется в функциональном виде, а именно для вариационных производных решения задачи $\xi(\mathbf{R}, t)$ и $\mathbf{p}(\mathbf{R}, t)$ имеют место соотношения

$$
\begin{aligned}
& \frac{\delta \xi(\mathbf{R}, t)}{\delta \mathbf{u}\left(\mathbf{R}^{\prime}, z^{\prime}, t^{\prime}\right)} \sim \theta\left(z^{\prime}-H(\mathbf{R})\right) \theta\left(t-t^{\prime}\right), \\
& \frac{\delta \mathbf{p}(\mathbf{R}, t)}{\delta \mathbf{u}\left(\mathbf{R}^{\prime}, z^{\prime}, t^{\prime}\right)} \sim \theta\left(z^{\prime}-H(\mathbf{R})\right) \theta\left(t-t^{\prime}\right),
\end{aligned}
$$

где $\theta(z)$ - тета-функция Хевисайда.

Выведем уравнение Лиувилля для рассматриваемой динамической системы. Общая методика вывода подобных уравнений подробно изложена в монографиях [2], [3].

\section{3. УРАВНЕНИЕ ЛИУВИЛЛЯ}

Введем совместную индикаторную функцию смещения поверхности и ее градиента:

$$
\varphi(\mathbf{R}, t ; \xi, \mathbf{p})=\delta(\xi(\mathbf{R}, t)-\xi) \delta(\mathbf{p}(\mathbf{R}, t)-\mathbf{p}) .
$$

Дифференцируя это выражение по времени, получаем равенство

$$
\frac{\partial \varphi(\mathbf{R}, t ; \xi, \mathbf{p})}{\partial t}=-\frac{\partial \xi(\mathbf{R}, t)}{\partial t} \frac{\partial \varphi(\mathbf{R}, t ; \xi, \mathbf{p})}{\partial \xi}-\frac{\partial p_{\beta}(\mathbf{R}, t)}{\partial t} \frac{\partial \varphi(\mathbf{R}, t ; \xi, \mathbf{p})}{\partial p_{\beta}}
$$

которое с учетом исходных динамических уравнений (4) и (5) можно записать в виде незамкнутого уравнения

$$
\begin{aligned}
\frac{\partial \varphi(\mathbf{R}, t ; \xi, \mathbf{p})}{\partial t}= & \frac{\partial}{\partial \xi}\left[u_{\alpha}(\mathbf{R}, \xi, t) p_{\alpha}-w(\mathbf{R}, \xi ; t)\right] \varphi(\mathbf{R}, t ; \xi, \mathbf{p})+ \\
& +\frac{\partial}{\partial p_{\beta}}\left[\frac{\partial u_{\alpha}(\mathbf{R}, \xi ; t)}{\partial R_{\beta}} p_{\alpha}+\frac{\partial u_{\alpha}(\mathbf{R}, \xi ; t)}{\partial \xi} p_{\beta} p_{\alpha}\right] \varphi(\mathbf{R}, t ; \xi, \mathbf{p})-
\end{aligned}
$$




$$
\begin{aligned}
& -\frac{\partial}{\partial p_{\beta}}\left[\frac{\partial w(\mathbf{R}, \xi ; t)}{\partial R_{\beta}}+\frac{\partial w(\mathbf{R}, \xi ; t)}{\partial \xi} p_{\beta}\right] \varphi(\mathbf{R}, t ; \xi, \mathbf{p})+ \\
& +\frac{\partial^{2} \xi(\mathbf{R}, t)}{\partial R_{\beta} \partial R_{\alpha}} u_{\alpha}(\mathbf{R}, \xi ; t) \frac{\partial}{\partial p_{\beta}} \varphi(\mathbf{R}, t ; \xi, \mathbf{p}),
\end{aligned}
$$

содержащего уже вторую пространственную производную функции смещения водной поверхности.

Для замыкания данного уравнения вычислим производную $\frac{\partial \varphi(\mathbf{R}, t ; \xi, \mathbf{p})}{\partial R_{\alpha}}$. Для нее получаем выражение

$$
-\left(\frac{\partial}{\partial R_{\alpha}}+p_{\alpha} \frac{\partial}{\partial \xi}\right) \varphi(\mathbf{R}, t ; \xi, \mathbf{p})=\frac{\partial^{2} \xi(\mathbf{R}, t)}{\partial R_{\alpha} \partial R_{\beta}} \frac{\partial}{\partial p_{\beta}} \varphi(\mathbf{R}, t ; \xi, \mathbf{p}),
$$

с помощью которого можно исключить последний член в уравнении (9). В результате получаем замкнутое уравнение Лиувилля

$$
\begin{aligned}
\frac{\partial \varphi(\mathbf{R}, t ; \xi, \mathbf{p})}{\partial t}= & -u_{\alpha}(\mathbf{R}, \xi ; t)\left(\frac{\partial}{\partial R_{\alpha}}+p_{\alpha} \frac{\partial}{\partial \xi}\right) \varphi(\mathbf{R}, t ; \xi, \mathbf{p})+ \\
& +\frac{\partial}{\partial \xi}\left[u_{\alpha}(\mathbf{R}, \xi ; t) p_{\alpha}-w(\mathbf{R}, \xi ; t)\right] \varphi(\mathbf{R}, t ; \xi, \mathbf{p})+ \\
& +\frac{\partial}{\partial p_{\beta}}\left(\frac{\partial u_{\alpha}(\mathbf{R}, \xi ; t)}{\partial R_{\beta}} p_{\alpha}+\frac{\partial u_{\alpha}(\mathbf{R}, \xi ; t)}{\partial \xi} p_{\beta} p_{\alpha}\right) \varphi(\mathbf{R}, t ; \xi, \mathbf{p})- \\
& -\frac{\partial}{\partial p_{\beta}}\left(\frac{\partial w(\mathbf{R}, \xi ; t)}{\partial R_{\beta}}+\frac{\partial w(\mathbf{R}, \xi ; t)}{\partial \xi} p_{\beta}\right) \varphi(\mathbf{R}, t ; \xi, \mathbf{p})
\end{aligned}
$$

с начальным условием $\varphi(\mathbf{R}, 0 ; \xi, \mathbf{p})=\delta\left(\xi-\xi_{0}(\mathbf{R})\right) \delta\left(\mathbf{p}-\mathbf{p}_{0}(\mathbf{R})\right)$.

Отметим, что уравнение (11) описывает совместную плотность вероятностей для смещения поверхности и его пространственного градиента при детерминированных параметрах динамической системы и при случайных начальных условиях. Нас интересует случай генерации морского волнения случайным гидродинамическим полем скоростей $\mathbf{u}(\mathbf{r}, t)$, которое мы будем считать случайным гауссовым полем, статистически однородным и изотропным в пространстве и статистически стационарным во времени с корреляционной и спектральными функциями

$$
\begin{aligned}
B_{i j}\left(\mathbf{r}-\mathbf{r}^{\prime}, t-t^{\prime}\right) & =\left\langle u_{i}(\mathbf{r}, t) u_{i}\left(\mathbf{r}^{\prime}, t^{\prime}\right)\right\rangle=\int d \mathbf{k} E_{i j}\left(k, t-t^{\prime}\right) e^{\mathrm{i} \mathbf{k}\left(\mathbf{r}-\mathbf{r}^{\prime}\right)}, \\
E_{i j}(k, t) & =\frac{1}{(2 \pi)^{3}} \int d \mathbf{r} B_{i j}(\mathbf{r}, t) e^{-\mathrm{i} \mathbf{k r}} .
\end{aligned}
$$

Нас интересуют два примера случайного поля скоростей: несжимаемая гидродинамическая турбулентность и случайные волновые гидродинамические поля. В первом случае спектральная функция имеет вид

$$
E_{i j}(k, t)=E(k, t)\left(\delta_{i j}-\frac{k_{i} k_{j}}{k^{2}}\right),
$$

во втором случае корреляционная функция поля скорости задается как

$$
B_{i j}(\mathbf{r}, t)=\int d \mathbf{k} E_{i j}(\mathbf{k}) e^{-\lambda k^{2} t} \cos (\mathbf{k r}-\omega(\mathbf{k}) t)
$$


где функция $E_{i j}(\mathbf{k})$ такова, что $\int d \mathbf{k} E_{i i}(\mathbf{k})=\sigma_{\mathbf{u}}^{2}-$ дисперсия поля скорости, функция $\omega=\omega(\mathbf{k})>0$ определяет дисперсионную кривую для волновых движений, а параметр $\lambda$ описывает затухание волн. По-видимому, первый случай описывает неподвижные структурообразования типа представленных на рис. 1, а вторая корреляционная функция описывает распространяющиеся структуры волнового типа.

В обоих случаях совместная плотность вероятностей смещения морской поверхности и его градиента является индикаторной функцией (7), усредненной по ансамблю реализаций случайного поля $\mathbf{u}(\mathbf{r}, t)$ :

$$
P(\mathbf{R}, t ; \xi, \mathbf{p})=\langle\varphi(\mathbf{R}, t ; \xi, \mathbf{p})\rangle_{\mathbf{u}}
$$

При этом размыкание корреляции поля скорости $\mathbf{u}(\mathbf{r}, t)$ с произвольным функционалом от него осуществляется на основе формулы Фурутцу-Новикова (см., например, монографии [2], [3]) с учетом топографических неоднородностей морского дна, заданных функцией $H(\mathbf{R})$ (см. рис. 2 и выражения (6)):

$$
\begin{aligned}
& \left\langle u_{i}(\mathbf{R}, \xi, t) R[\mathbf{u}(\widetilde{\mathbf{R}}, \widetilde{\xi}, \tau)\rangle_{\mathbf{u}}=\right. \\
& \quad=\int d \mathbf{R}^{\prime} \int_{H(\mathbf{R})}^{\xi+0} d \xi^{\prime} \int_{0}^{t} d t^{\prime} B_{i j}\left(\mathbf{R}-\mathbf{R}^{\prime}, \xi-\xi^{\prime}, t-t^{\prime}\right)\left\langle\frac{\delta R[\mathbf{u}(\widetilde{\mathbf{R}}, \tilde{z}, \tau)]}{\delta u_{j}\left(\mathbf{R}^{\prime}, \xi^{\prime}, t^{\prime}\right)}\right\rangle_{\mathbf{u}} .
\end{aligned}
$$

Это соотношение можно переписать в виде

$$
\begin{aligned}
\left\langle u_{i}(\mathbf{R}, \xi, t) R[\mathbf{u}(\widetilde{\mathbf{R}}, \widetilde{\xi}, \tau)]\right\rangle_{\mathbf{u}}=\int d \mathbf{R}^{\prime} \int_{-\infty}^{\xi+0} d \xi^{\prime} \theta\left(\xi^{\prime}-H(\mathbf{R})\right) \times \\
\times \int_{0}^{t} d t^{\prime} B_{i j}\left(\mathbf{R}-\mathbf{R}^{\prime}, \xi-\xi^{\prime}, t-t^{\prime}\right)\left\langle\frac{\delta R[\mathbf{u}(\widetilde{\mathbf{R}}, \tilde{z}, \tau)]}{\delta u_{j}\left(\mathbf{R}^{\prime}, \xi^{\prime}, t^{\prime}\right)}\right\rangle_{\mathbf{u}} .
\end{aligned}
$$

Предполагая, что топография дна также задана статистически со средним значением $\langle H(\mathbf{R})\rangle=-H$, где $H-$ средняя глубина моря, мы должны усреднить формулу (14) по ансамблю ее реализаций. Считая при этом, что топографические неоднородности дна статистически не зависят от гидродинамического поля скоростей, получаем выражение

$$
\begin{aligned}
\left\langle u_{i}(\mathbf{R}, \xi, t) R[\mathbf{u}(\widetilde{\mathbf{R}}, \widetilde{\xi}, \tau)]\right\rangle_{\mathbf{u}}=\int d \mathbf{R}^{\prime} \int_{-\infty}^{\xi+0} d \xi^{\prime}\left\langle\theta\left(\xi^{\prime}-H(\mathbf{R})\right)\right\rangle_{H} \times \\
\times \int_{0}^{t} d t^{\prime} B_{i j}\left(\mathbf{R}-\mathbf{R}^{\prime}, \xi-\xi^{\prime}, t-t^{\prime}\right)\left\langle\frac{\delta R[\mathbf{u}(\widetilde{\mathbf{R}}, \tilde{z}, \tau)]}{\delta u_{j}\left(\mathbf{R}^{\prime}, \xi^{\prime}, t^{\prime}\right)}\right\rangle_{\mathbf{u}},
\end{aligned}
$$

где функция $\langle\theta(\xi-H(\mathbf{R}))\rangle_{H}$ является интегральной функцией распределения вероятностей топографических неоднородностей $H(\mathbf{R})$, т. е. вероятностью события $\xi>H(\mathbf{R})$. Для статистического однородного случайного поля $H(\mathbf{R})$ эта функция не зависит, естественно, от пространственной точки $\mathbf{R}$ и, следовательно,

$$
\langle\theta(\xi-H(\mathbf{R}))\rangle_{H}=\mathrm{P}\{\xi>H(\mathbf{R})\}=\mathrm{P}(H ; \xi) .
$$

Отметим, что для бесконечно глубокого моря (при $H \rightarrow \infty$ на рис. 2) мы имеем $\mathrm{P}(H ; \xi) \rightarrow 1$. 
В общем случае в рамках диффузионного приближения (см., например, монографии [2], [3]) формула (15) упрощается и принимает вид

$$
\begin{aligned}
& \left\langle u_{i}(\mathbf{R}, \xi, t) R[\mathbf{u}(\widetilde{\mathbf{R}}, \widetilde{\xi}, \tau)]\right\rangle_{\mathbf{u}}=\int d \mathbf{R}^{\prime} \int_{-\infty}^{\xi+0} d \xi^{\prime} \mathrm{P}\left(H ; \xi^{\prime}\right) \times \\
& \quad \times B_{i j}\left(\mathbf{R}-\mathbf{R}^{\prime}, \xi-\xi^{\prime}\right)\left\langle\frac{\delta R[\mathbf{u}(\widetilde{\mathbf{R}}, \tilde{z}, \tau)]}{\delta u_{j}\left(\mathbf{R}^{\prime}, \xi^{\prime}, t-0\right)}\right\rangle_{\mathbf{u}}, \quad B_{i j}(\mathbf{R}, \xi)=\int_{0}^{\infty} d \tau B_{i j}(\mathbf{R}, \xi, \tau) .
\end{aligned}
$$

В частности,

$$
\begin{aligned}
\left\langle u_{\alpha}(\mathbf{R}, \xi, t) \varphi[\mathbf{u}(\widetilde{\mathbf{R}}, \widetilde{\xi}, \tau)]\right\rangle_{\mathbf{u}}= & \\
= & \int d \mathbf{R}^{\prime} \int_{-\infty}^{\xi+0} d \xi^{\prime} \mathrm{P}\left(H ; \xi^{\prime}\right) B_{\alpha \beta}\left(\mathbf{R}-\mathbf{R}^{\prime}, \xi-\xi^{\prime}\right)\left\langle\frac{\delta \varphi[\mathbf{u}(\widetilde{\mathbf{R}}, \tilde{z}, \tau)]}{\delta u_{\beta}\left(\mathbf{R}^{\prime}, \xi^{\prime}, t-0\right)}\right\rangle_{\mathbf{u}}+ \\
& +\int d \mathbf{R}^{\prime} \int_{-\infty}^{\xi+0} d \xi^{\prime} \mathrm{P}\left(H ; \xi^{\prime}\right) B_{\alpha w}\left(\mathbf{R}-\mathbf{R}^{\prime}, \xi-\xi^{\prime}\right)\left\langle\frac{\delta \varphi[\mathbf{u}(\widetilde{\mathbf{R}}, \tilde{z}, \tau)]}{\delta w\left(\mathbf{R}^{\prime}, \xi^{\prime}, t-0\right)}\right\rangle_{\mathbf{u}} \\
\langle w(\mathbf{R}, \xi, t) \varphi[\mathbf{u}(\widetilde{\mathbf{R}}, \widetilde{\xi}, \tau)]\rangle_{\mathbf{u}}= & \\
= & \int d \mathbf{R}^{\prime} \int_{-\infty}^{\xi+0} d \xi^{\prime} \mathrm{P}\left(H ; \xi^{\prime}\right) B_{w \beta}\left(\mathbf{R}-\mathbf{R}^{\prime}, \xi-\xi^{\prime}\right)\left\langle\frac{\delta \varphi[\mathbf{u}(\widetilde{\mathbf{R}}, \tilde{z}, \tau)]}{\delta u_{\beta}\left(\mathbf{R}^{\prime}, \xi^{\prime}, t-0\right)}\right\rangle_{\mathbf{u}}+ \\
& +\int d \mathbf{R}^{\prime} \int_{-\infty}^{\xi+0} \mathrm{P}\left(H ; \xi^{\prime}\right) d \xi B_{w w}\left(\mathbf{R}-\mathbf{R}^{\prime}, \xi-\xi^{\prime}\right)\left\langle\frac{\delta \varphi[\mathbf{u}(\widetilde{\mathbf{R}}, \tilde{z}, \tau)]}{\delta w\left(\mathbf{R}^{\prime}, \xi^{\prime}, t-0\right)}\right\rangle_{\mathbf{u}}
\end{aligned}
$$

и уравнение для плотности вероятностей (12) принимает вид

$$
\begin{aligned}
& \frac{\partial P(\mathbf{R}, t ; \xi, \mathbf{p})}{\partial t}= \\
& =-\int d \mathbf{R}^{\prime} \int_{-\infty}^{\xi+0} d \xi^{\prime} \mathrm{P}\left(H ; \xi^{\prime}\right) B_{\alpha \beta}\left(\mathbf{R}-\mathbf{R}^{\prime}, \xi-\xi^{\prime}\right)\left(\frac{\partial}{\partial R_{\alpha}}+p_{\alpha} \frac{\partial}{\partial \xi}\right)\left\langle\frac{\delta \varphi[\mathbf{u}(\widetilde{\mathbf{R}}, \tilde{z}, \tau)]}{\delta u_{\beta}\left(\mathbf{R}^{\prime}, \xi^{\prime}, t-0\right)}\right\rangle- \\
& \quad-\int d \mathbf{R}^{\prime} \int_{-\infty}^{\xi+0} d \xi^{\prime} \mathrm{P}\left(H ; \xi^{\prime}\right) B_{\alpha w}\left(\mathbf{R}-\mathbf{R}^{\prime}, \xi-\xi^{\prime}\right)\left(\frac{\partial}{\partial R_{\alpha}}+p_{\alpha} \frac{\partial}{\partial \xi}\right)\left\langle\frac{\delta \varphi[\mathbf{u}(\widetilde{\mathbf{R}}, \tilde{z}, \tau)]}{\delta w\left(\mathbf{R}^{\prime}, \xi^{\prime}, t-0\right)}\right\rangle+ \\
& \quad+\frac{\partial}{\partial \xi} \int d \mathbf{R}^{\prime} \int_{-\infty}^{\xi+0} d \xi^{\prime} \mathrm{P}\left(H ; \xi^{\prime}\right) B_{\alpha \beta}\left(\mathbf{R}-\mathbf{R}^{\prime}, \xi-\xi^{\prime}\right) p_{\alpha}\left\langle\frac{\delta \varphi[\mathbf{u}(\widetilde{\mathbf{R}}, \tilde{z}, \tau)]}{\delta u_{\beta}\left(\mathbf{R}^{\prime}, \xi^{\prime}, t-0\right)}\right\rangle+ \\
& +\frac{\partial}{\partial \xi} \int d \mathbf{R}^{\prime} \int_{-\infty}^{\xi+0} d \xi^{\prime} \mathrm{P}\left(H ; \xi^{\prime}\right) B_{\alpha w}\left(\mathbf{R}-\mathbf{R}^{\prime}, \xi-\xi^{\prime}\right) p_{\alpha}\left\langle\frac{\delta \varphi[\mathbf{u}(\widetilde{\mathbf{R}}, \tilde{z}, \tau)]}{\delta w\left(\mathbf{R}^{\prime}, \xi^{\prime}, t-0\right)}\right\rangle- \\
& \quad-\frac{\partial}{\partial \xi} \int d \mathbf{R}^{\prime} \int_{-\infty}^{\xi+0} d \xi^{\prime} \mathrm{P}\left(H ; \xi^{\prime}\right) B_{w \beta}\left(\mathbf{R}-\mathbf{R}^{\prime}, \xi-\xi^{\prime}\right)\left\langle\frac{\delta \varphi[\mathbf{u}(\widetilde{\mathbf{R}}, \tilde{z}, \tau]}{\delta u_{\beta}\left(\mathbf{R}^{\prime}, \xi^{\prime}, t-0\right)}\right\rangle- \\
& \quad-\frac{\partial}{\partial \xi} \int d \mathbf{R}^{\prime} \int_{-\infty}^{\xi+0} d \xi^{\prime} \mathrm{P}\left(H ; \xi^{\prime}\right) B_{w w}\left(\mathbf{R}-\mathbf{R}^{\prime}, \xi-\xi^{\prime}\right)\left\langle\frac{\delta \varphi[\mathbf{u}(\widetilde{\mathbf{R}}, \tilde{z}, \tau]}{\delta w\left(\mathbf{R}^{\prime}, \xi^{\prime}, t-0\right)}\right\rangle+ \\
& +\int d \mathbf{R}^{\prime} \int_{-\infty}^{\xi+0} d \xi^{\prime} \mathrm{P}\left(H ; \xi^{\prime}\right) \frac{\partial B_{\alpha \beta}\left(\mathbf{R}-\mathbf{R}^{\prime}, \xi-\xi^{\prime}\right)}{\partial R_{\gamma}} \frac{\partial}{\partial p_{\gamma}} p_{\alpha}\left\langle\frac{\delta \varphi[\mathbf{u}(\widetilde{\mathbf{R}}, \tilde{z}, \tau)]}{\delta u_{\beta}\left(\mathbf{R}^{\prime}, \xi^{\prime}, t-0\right)}\right\rangle+
\end{aligned}
$$




$$
\begin{aligned}
& +\int d \mathbf{R}^{\prime} \int_{-\infty}^{\xi+0} d \xi^{\prime} \mathrm{P}\left(H ; \xi^{\prime}\right) \frac{\partial B_{\alpha w}\left(\mathbf{R}-\mathbf{R}^{\prime}, \xi-\xi^{\prime}\right)}{\partial R_{\gamma}} \frac{\partial}{\partial p_{\gamma}} p_{\alpha}\left\langle\frac{\delta \varphi[\mathbf{u}(\widetilde{\mathbf{R}}, \tilde{z}, \tau)]}{\delta w\left(\mathbf{R}^{\prime}, \xi^{\prime}, t-0\right)}\right\rangle+ \\
& +\int d \mathbf{R}^{\prime} \int_{-\infty}^{\xi+0} d \xi^{\prime} \mathrm{P}\left(H ; \xi^{\prime}\right) \frac{\partial B_{\alpha \beta}\left(\mathbf{R}-\mathbf{R}^{\prime}, \xi-\xi^{\prime}\right)}{\partial \xi} \frac{\partial}{\partial p_{\gamma}} p_{\gamma} p_{\alpha}\left\langle\frac{\delta \varphi[\mathbf{u}(\widetilde{\mathbf{R}}, \tilde{z}, \tau)]}{\delta u_{\beta}\left(\mathbf{R}^{\prime}, \xi^{\prime}, t-0\right)}\right\rangle+ \\
& +\int d \mathbf{R}^{\prime} \int_{-\infty}^{\xi+0} d \xi^{\prime} \mathrm{P}\left(H ; \xi^{\prime}\right) \frac{\partial B_{\alpha w}\left(\mathbf{R}-\mathbf{R}^{\prime}, \xi-\xi^{\prime}\right)}{\partial \xi} \frac{\partial}{\partial p_{\gamma}} p_{\gamma} p_{\alpha}\left\langle\frac{\delta \varphi[\mathbf{u}(\widetilde{\mathbf{R}}, \tilde{z}, \tau]}{\delta w\left(\mathbf{R}^{\prime}, \xi^{\prime}, t-0\right)}\right\rangle- \\
& -\int d \mathbf{R}^{\prime} \int_{-\infty}^{\xi+0} d \xi^{\prime} \mathrm{P}\left(H ; \xi^{\prime}\right) \frac{\partial B_{w \beta}\left(\mathbf{R}-\mathbf{R}^{\prime}, \xi-\xi^{\prime}\right)}{\partial R_{\beta}} \frac{\partial}{\partial p_{\beta}}\left\langle\frac{\delta \varphi[\mathbf{u}(\widetilde{\mathbf{R}}, \tilde{z}, \tau]}{\delta u_{\beta}\left(\mathbf{R}^{\prime}, \xi^{\prime}, t-0\right)}\right\rangle- \\
& -\int d \mathbf{R}^{\prime} \int_{-\infty}^{\xi+0} d \xi^{\prime} \mathrm{P}\left(H ; \xi^{\prime}\right) \frac{\partial B_{w w}\left(\mathbf{R}-\mathbf{R}^{\prime}, \xi-\xi^{\prime}\right)}{\partial R_{\beta}} \frac{\partial}{\partial p_{\beta}}\left\langle\frac{\delta \varphi[\mathbf{u}(\widetilde{\mathbf{R}}, \tilde{z}, \tau)]}{\delta w\left(\mathbf{R}^{\prime}, \xi^{\prime}, t-0\right)}\right\rangle- \\
& -\int d \mathbf{R}^{\prime} \int_{-\infty}^{\xi+0} d \xi^{\prime} \mathrm{P}\left(H ; \xi^{\prime}\right) \frac{\partial B_{w \beta}\left(\mathbf{R}-\mathbf{R}^{\prime}, \xi-\xi^{\prime}\right)}{\partial \xi} \frac{\partial}{\partial p_{\beta}} p_{\beta}\left\langle\frac{\delta \varphi[\mathbf{u}(\widetilde{\mathbf{R}}, \tilde{z}, \tau)]}{\delta u_{\beta}\left(\mathbf{R}^{\prime}, \xi^{\prime}, t-0\right)}\right\rangle- \\
& -\int d \mathbf{R}^{\prime} \int_{-\infty}^{\xi+0} d \xi^{\prime} \mathrm{P}\left(H ; \xi^{\prime}\right) \frac{\partial B_{w w}\left(\mathbf{R}-\mathbf{R}^{\prime}, \xi-\xi^{\prime}\right)}{\partial \xi} \frac{\partial}{\partial p_{\beta}} p_{\beta}\left\langle\frac{\delta \varphi[\mathbf{u}(\widetilde{\mathbf{R}}, \tilde{z}, \tau)]}{\delta w\left(\mathbf{R}^{\prime}, \xi^{\prime}, t-0\right)}\right\rangle .
\end{aligned}
$$

Записывая уравнение Лиувилля (11) в виде интегрального уравнения, мы получаем выражения для соответствующих вариационных производных (методику вывода см., например, в монографиях [2], [3]):

$$
\begin{aligned}
& \left\langle\frac{\delta \varphi[\mathbf{u}(\widetilde{\mathbf{R}}, \tilde{z}, \tau)]}{\delta u_{\beta}\left(\mathbf{R}^{\prime}, \xi^{\prime}, t-0\right)}\right\rangle_{\mathbf{u}}=-\delta\left(\mathbf{R}-\mathbf{R}^{\prime}\right) \delta\left(\xi-\xi^{\prime}\right)\left(\frac{\partial}{\partial R_{\beta}}+p_{\beta} \frac{\partial}{\partial \xi}\right) P(\mathbf{R}, t ; \xi, \mathbf{p}+ \\
& +\frac{\partial}{\partial \xi} \delta\left(\mathbf{R}-\mathbf{R}^{\prime}\right) \delta\left(\xi-\xi^{\prime}\right) p_{\beta} P(\mathbf{R}, t ; \xi, \mathbf{p})+ \\
& \quad+\frac{\partial}{\partial p_{\gamma}} \frac{\partial \delta\left(\mathbf{R}-\mathbf{R}^{\prime}\right)}{\partial R_{\gamma}} \delta\left(\xi-\xi^{\prime}\right) p_{\beta} P(\mathbf{R}, t ; \xi, \mathbf{p})+ \\
& +\frac{\partial}{\partial p_{\gamma}} \delta\left(\mathbf{R}-\mathbf{R}^{\prime}\right) \frac{\partial \delta\left(\xi-\xi^{\prime}\right)}{\partial \xi} p_{\gamma} p_{\beta} P(\mathbf{R}, t ; \xi, \mathbf{p}), \\
& \left\langle\frac{\delta \varphi[\mathbf{u}(\widetilde{\mathbf{R}}, \tilde{z}, \tau)]}{\delta w\left(\mathbf{R}^{\prime}, \xi^{\prime}, t-0\right)}\right\rangle_{\mathbf{u}}=-\frac{\partial}{\partial \xi} \delta\left(\mathbf{R}-\mathbf{R}{ }^{\prime}\right) \delta\left(\xi-\xi^{\prime}\right) P(\mathbf{R}, t ; \xi, \mathbf{p})+ \\
& -\frac{\partial}{\partial p_{\gamma}} \frac{\partial \delta\left(\mathbf{R}-\mathbf{R}^{\prime}\right)}{\partial R_{\gamma}} \delta\left(\xi-\xi^{\prime}\right) P(\mathbf{R}, t ; \xi, \mathbf{p})- \\
& -\frac{\partial}{\partial p_{\gamma}} \delta\left(\mathbf{R}-\mathbf{R}^{\prime}\right) \frac{\partial \delta\left(\xi-\xi^{\prime}\right)}{\partial \xi} p_{\gamma} P(\mathbf{R}, t ; \xi, \mathbf{p}) .
\end{aligned}
$$

Отметим, что для однородных начальных условий $\xi_{0}(\mathbf{R})=0$ и $\mathbf{p}_{0}(\mathbf{R})=0$ все одноточечные статистические характеристики не зависят от пространственной координаты R, следовательно,

$$
P(\mathbf{R}, t ; \xi, \mathbf{p}) \equiv P(t ; \xi, \mathbf{p}) .
$$


В этом случае формулы (17) также упрощаются и принимают вид

$$
\begin{aligned}
& \left\langle\frac{\delta \varphi[\mathbf{u}(\widetilde{\mathbf{R}}, \tilde{z}, \tau)]}{\delta u_{\beta}\left(\mathbf{R}^{\prime}, \xi^{\prime}, t-0\right)}\right\rangle_{\mathbf{u}}=-\delta\left(\mathbf{R}-\mathbf{R}^{\prime}\right) \delta\left(\xi-\xi^{\prime}\right) p_{\beta} \frac{\partial}{\partial \xi} P(t ; \xi, \mathbf{p})+ \\
& \quad+\frac{\partial}{\partial \xi} \delta\left(\mathbf{R}-\mathbf{R}^{\prime}\right) \delta\left(\xi-\xi^{\prime}\right) p_{\beta} P(t ; \xi, \mathbf{p})+\frac{\partial}{\partial p_{\gamma}} \frac{\partial \delta\left(\mathbf{R}-\mathbf{R}^{\prime}\right)}{\partial R_{\gamma}} \delta\left(\xi-\xi^{\prime}\right) p_{\beta} P(t ; \xi, \mathbf{p})+ \\
& \quad+\frac{\partial}{\partial p_{\gamma}} \delta\left(\mathbf{R}-\mathbf{R}^{\prime}\right) \frac{\partial \delta\left(\xi-\xi^{\prime}\right)}{\partial \xi} p_{\gamma} p_{\beta} P(t ; \xi, \mathbf{p}), \\
& \left\langle\frac{\delta \varphi[\mathbf{u}(\widetilde{\mathbf{R}}, \tilde{z}, \tau)]}{\delta w\left(\mathbf{R}^{\prime}, \xi^{\prime}, t-0\right)}\right\rangle_{\mathbf{u}}=-\frac{\partial}{\partial \xi} \delta\left(\mathbf{R}-\mathbf{R}^{\prime}\right) \delta\left(\xi-\xi^{\prime}\right) P(t ; \xi, \mathbf{p})+ \\
& -\frac{\partial}{\partial p_{\gamma}} \frac{\partial \delta\left(\mathbf{R}-\mathbf{R}^{\prime}\right)}{\partial R_{\gamma}} \delta\left(\xi-\xi^{\prime}\right) P(t ; \xi, \mathbf{p})-\frac{\partial}{\partial p_{\gamma}} \delta\left(\mathbf{R}-\mathbf{R}^{\prime}\right) \frac{\partial \delta\left(\xi-\xi^{\prime}\right)}{\partial \xi} p_{\gamma} P(t ; \xi, \mathbf{p}) .
\end{aligned}
$$

Отметим также, что в этом случае уравнение для функции $P(t ; \xi, \mathbf{p})$ можно упростить и перейти к плотности вероятностей скалярных величин $P(t ; \xi, p)$, где $p=|\mathbf{p}|$.

Описать пространственное структурообразование случайного поля $\xi(\mathbf{R}, t)$ в отдельных реализациях можно на основе идей статистической топографии [2], [3], $[5]-[8]$.

\section{4. СТАТИСТИЧЕСКАЯ ТОПОГРАФИЯ СЛУЧАЙНОГО ПОЛЯ $\xi(\mathbf{R}, t)$}

В статистической топографии случайных полей основным объектом изучения, как и в обычной топографии горных массивов, является система контуров - линий уровня постоянных значений, определяемых равенством $\xi(\mathbf{R}, t)=h=$ const. Для анализа системы контуров удобно рассмотреть индикаторную функцию

$$
\varphi(\mathbf{R}, t ; \xi)=\delta(\xi(\mathbf{R}, t)-\xi)
$$

Через эту функцию выражаются, например, такие величины, как общая площадь, ограниченная линиями уровня областей (общая площадь “подошвы” волн), в которых случайное поле $\xi(\mathbf{R}, t)$ превышает заданный уровень $h$, т. е. $\xi(\mathbf{R}, t)>h$,

$$
S(t ; h)=\int d \mathbf{R} \theta(\xi(\mathbf{R}, t)-h)=\int d \mathbf{R} \int_{h}^{\infty} d \xi \varphi(\mathbf{R}, t ; \xi),
$$

и объем водной массы, заключенный в этих областях,

$$
V(t ; h)=\int d \mathbf{R} \xi(\mathbf{R}, t) \theta(\xi(\mathbf{R}, t)-h)=\int d \mathbf{R} \int_{h}^{\infty} d \xi \xi \varphi(\mathbf{R}, t ; \xi) .
$$

Среднее значение индикаторной функции по ансамблю реализаций случайного поля $\xi(\mathbf{R}, t)$ определяет одновременну́ю и одноточечную плотность вероятностей

$$
P(\mathbf{R}, t ; \xi)=\langle\delta(\xi(\mathbf{R}, t)-\xi)\rangle
$$


следовательно, средние по ансамблю реализаций значения величин $S(t ; h)$ и $V(t ; h)$ определяются непосредственно этой плотностью вероятностей:

$$
\begin{aligned}
\langle S(t ; h)\rangle & =\int d \mathbf{R} \int_{h}^{\infty} d \xi P(\mathbf{R}, t ; \xi), \\
\langle V(t ; h)\rangle & =\int d \mathbf{R} \int_{h}^{\infty} d \xi \xi P(\mathbf{R}, t ; \xi) .
\end{aligned}
$$

Обсудим теперь условия возникновения стохастического структурообразования для больших случайных волн $\xi(\mathbf{R}, t)$. Ясно, что для положительного поля $\xi(\mathbf{R}, t)$ необходимым условием является условие малости общей площади "подошвы" волн $\langle S(t ; h)\rangle$ при эволюции системы во времени. Но аномальная волна будет образовываться, только если на этой площади будет сосредоточен достаточно большой объем водной массы $\langle V(t ; h)\rangle$. При этом характерная высота таких волн будет определяться величиной

$$
H_{\text {wave }}(t ; h) \sim \frac{\langle V(t ; h)\rangle}{\langle S(t ; h)\rangle} .
$$

Увеличивая параметр $h$, можно определить как максимальную высоту волны, так и время ее существования.

Для пространственно-однородного поля $\xi(\mathbf{R}, t)$ одноточечная плотность вероятностей $P(\mathbf{R}, t ; \xi)$ не зависит от $\mathbf{R}$. В этом случае статистические средние всех выражений (без интегрирования по $\mathbf{R}$ ) будут описывать удельные (приходящиеся на единицу площади) значения этих величин. Так, удельная средняя площадь “подошвы" волны $\left\langle s_{\text {wave }}(t ; h)\right\rangle$, на которой случайное поле $\xi(\mathbf{R}, t)$ превышает заданный уровень $h$, совпадает с вероятностью события $\xi(\mathbf{R}, t)>h$ в любой точке пространства:

$$
\left\langle s_{\text {wave }}(t ; h)\right\rangle=\langle\theta(\xi(\mathbf{R}, t)-h)\rangle=\mathrm{P}\{\xi(\mathbf{R}, t)>h\},
$$

и средняя удельная площадь является геометрической интерпретацией вероятности события $\xi(\mathbf{R}, t)>h$, не зависящей, естественно, от точки $\mathbf{R}$. Таким образом, ясно, что экстремально большие волны могут порождаться только очень маловероятными событиями, которые, однако, всегда присутствуют в случайной среде. Но такие волны будут возникать только при условии, что параметр

$$
h_{\text {wave }}(t ; h) \sim \frac{\left\langle v_{\text {wave }}(t ; h)\right\rangle}{\left\langle s_{\text {wave }}(t ; h)\right\rangle}
$$

достаточно велик. Увеличивая параметр $h$, можно определить как максимальную высоту волны, так и время ее существования.

Таким образом, мы полностью сформулировали постановку статистической задачи. Чтобы ответить на поставленный в аннотации вопрос, необходимо далее подставить значения вариационных производных (17) или (18) в уравнение для плотности вероятностей (16), вычислить все интегралы и проанализировать получившееся уравнение с точки зрения статистической топографии.

Благодарности. Автор признателен О.И. Яковенко и М.А. Соколовскому за представленные фотографии, которые и послужили стимулом для написания настоящей работы. Автор также благодарен рецензенту за моральную поддержку. Работа выполнена при поддержке РФФИ (грант № 13-05-00004). 


\section{Список литературы}

[1] Секст Эмпирик, “Две книги против физиков", Сочинения в двух томах, т.1, ред. А. Ф. Лосев, Мысль, М., 1975, 244-376.

[2] В. И. Кляцкин, Динамика стохастических систем, Физматлит, М., 2002.

[3] В. И. Кляцкин, Очерки по динамике стохастических систем, УРСС, М., 2012.

[4] Я.Б. Зельдович, С. А. Молчанов, А. А. Рузмайкин, Д. Д. Соколов, УФН, 152:5 (1987), $3-32$.

[5] В. И. Кляцкин, УФН, 182:11 (2012), 1235-1237.

[6] V.I. Klyatskin, "On the criterion of stochastic structure formation in random media", Chaos and Complex Systems, Proceedings of the 4th International Interdisciplinary Chaos Symposium (Antalya, Turkey, April 29 - May 2, 2012), eds. S. G. Stavrinides, S. Banerjee, S. H. Caglar, M. Ozer, Springer, Berlin, 2013, 69-74.

[7] В. И. Кляцкин, ТМФ, 176:3 (2013), 494-512.

[8] V. I. Klyatskin, Russ. J. Math. Phys., 20:3 (2013), 295-314.

[9] В. И. Кляцкин, ПММ, 33:5 (1969), 889-891.

[10] В. И. Кляцкин, Изв. РАН. Физ. атм. и океана, 31:6 (1995), 749-754.

[11] V. Klyatskin, D. Gurarie, Phys. D, 98:2-4 (1996), 466-480.

[12] H. J. Hopfinger, F. K. Browand, Nature, 295 (1982), 393-394.

[13] B. M. Boubnov, G.S. Golitsyn, J. Fluid Mech., 167:6 (1986), 503-531.

[14] E. J. Hopfinger, "Turbulence and vortices in rotating fluids", Theoretical and Applied Mechanics (Saint-Martin-d'Heres, France, August 21-27, 1988), eds. P. Germain, M. Piau, D. Caillerie, North-Holland, Amsterdam, 1989, 117-138.

[15] B. M. Boubnov, G.S. Golitsyn, Convection in Rotating Fluids, Fluid Mechanics and Its Applications, 29, Kluwer, Dordrecht, 1995.

[16] V. I. Pavlov, D. Buisine, V.P. Goncharov, Nonlin. Proc. Geophys., 8 (2001), 9-19.

[17] S. Karimova, Adv. Space Res., 50:8 (2012), 1107-1124.

[18] А.А. Куркин, Е.Н. Пелиновский, Волны-убийцы: факты, теория и моделирование, ННТУ, Н. Новгород, 2004.

[19] C. Kharif, E. Pelinovskyy, A. Slyunaen, Rogue Waves in the Ocean, Springer, Berlin, 2009.

[20] В. Е. Захаров, Безумные волны, http://www.youtube.com/watch?v=Bt3ZOswGd-4, 2011; Волньь-убийць, http://www.zoomby.ru/watch/114832-academia, 2013.

[21] V. P. Ruban, Писъма в ЖЭТФ, 94:3 (2011), 194-198.

[22] В. П. Рубан, Писъма в ЖЭТФ, 95:9 (2012), 550-556.

[23] В. П. Рубан, Писъма в ЖЭТФ, 97:4 (2013), 215-220.

[24] В. П. Рубан, Писъма в ЖЭТФ, 97:12 (2013), 788-792.

Поступила в редакцию 12.02.2014, после доработки 4.03.2014 